\title{
Determining the healthfulness of an Irish Higher Education Institute (HEI) using the Healthy Campus Environment Audit (HCEA) tools
}

\author{
C. Murphy ${ }^{1}$, I. Martinez Yanes ${ }^{1}$ and C. Norton ${ }^{2,3,4}$ \\ ${ }^{1}$ Discipline of Dietetics, School of Allied Health, Faculty of Education and Health Sciences, University of Limerick, \\ Ireland, \\ ${ }^{2}$ Department of Physical Education \& Sport Sciences, Faculty of Education and Health Sciences, University of \\ Limerick, Limerick, Ireland, \\ ${ }^{3}$ Health Research Institute, University of Limerick, Limerick, Ireland and \\ ${ }^{4}$ Healthy UL
}

The transition from school to higher education is an important stage where lifelong health related habits are developed and anchored. Students' living and working conditions have been shown to positively influence the development of healthy habits including physical activity, healthy eating, alcohol consumption awareness and smoking awareness. Therefore, it is vital that HEIs support students by creating a healthy campus environment. Our aim was to evaluate wellness policies or initiatives, and perceptions of same, in an Irish HEI using validated HCEA 1 tools.

The POINTs audit is a validated tool used as an objective measure to determine the healthfulness of a campus environment ${ }^{(1)}$. It consists of 34 questions on health-promoting topics, and determines the existence of policies, initiatives, or the absence of same regarding health, well-being, and environmental aspects of the institution $(0=$ no initiative, $1=$ an initiative is in place, $2=\mathrm{a}$ policy is in place). A maximum score is 65 points. The College Environment Perception Survey (CEPS) ${ }^{(1)}$, is a subjective measure of the experience and opinion of students on the healthfulness of the campus. Furthermore, HEI stakeholder groups were consulted to verify findings for completeness and accuracy.

The POINTS audit awarded this HEI a score of 28 points. Two policies were identified regarding alcohol and smoking/vaping. Several initiatives were identified regarding healthy eating. There was no policies or initiatives in place regarding health education / nutrition / physical activity credit requirements, closed campus (no access by car), nutrient minimum/maximum standards, healthy food labels and point-of-purchase nutrition information, and food taxes and healthy food subsidies. Additionally, the absence of grow-your-own initiatives was noted. The CEPS survey was completed by 482 participants and identified a variety of perceptions on the healthfulness of the campus environment.

This investigation highlights the great efforts made by the HEI to promote healthy behaviour using initiatives, but it also identifies the lack of policies in place. The vital role policies and initiatives have in achieving health for all in a university setting is long established in the international Health Promoting Universities Framework ${ }^{(2)}$. These findings should provide a framework to take effective action by developing policies and initiatives. As it stands, only one HEI in the Republic of Ireland is officially recognised as a Health Promoting University. All HEIs should seek to achieve this or similar recognition to ensure they attain a campus environment that advocates optimal health and wellbeing for all.

\section{Acknowledgements}

The authors acknowledge the support of the Healthy UL Working Group and thank participants for their valued contributions

\section{References}

1. Horacek, et al. (2019) Int J Environ Res Public Health 4;16(5), 778.

2. Tsouros, et al. and WHO (1998) (No. EUR/ICP/CHVD 0309 01) Copenhagen: WHO Regional Office for Europe. 